Review Article

\title{
The Role of Toll-Like Receptor Signaling in the Progression of Heart Failure
}

\author{
Lili Yu $\mathbb{D}^{1,2,3,4}$ and Zhiwei Feng $\mathbb{D}^{1}$ \\ ${ }^{1}$ School of Basic Medical Sciences, Xinxiang Medical University, Xinxiang, Henan 453003, China \\ ${ }^{2}$ Pennington Biomedical Research Center, Louisiana State University, Baton Rouge, LA 70808, USA \\ ${ }^{3}$ Henan Key Laboratory of immunology and Targeted Drugs, Xinxiang, Henan 453003, China \\ ${ }^{4}$ Henan Collaborative Innovation Center of Molecular Diagnosis and Laboratory Medicine, Xinxiang, Henan 453003, China
}

Correspondence should be addressed to Lili Yu; merrys222@126.com and Zhiwei Feng; 123066@xxmu.edu.cn

Received 20 July 2017; Revised 28 November 2017; Accepted 14 December 2017; Published 8 February 2018

Academic Editor: Anshu Agrawal

Copyright ( 2018 Lili Yu and Zhiwei Feng. This is an open access article distributed under the Creative Commons Attribution License, which permits unrestricted use, distribution, and reproduction in any medium, provided the original work is properly cited.

\begin{abstract}
Medical systems worldwide are being faced with a growing need to understand mechanisms behind the pathogenesis of heart failure (HF) that is considered as a leading cause of morbidity and mortality around the world. Elevated levels of inflammatory mediators have been identified in patients with HF, which are primarily manifestations of innate immune responses mediated by pattern recognition receptors (PRRs). Toll-like receptors (TLRs), which belong to PRRs, are subjected to the release of pathogenassociated molecular patterns (PAMPs) and damage-associated molecular patterns (DAMPs) to generate innate immune responses. More and more emerging data indicate that TLR signaling pathway molecules are involved in the progression of HF. Herein, we present new data with regard to the activation of TLRs in the failing heart, focusing on TLR2, TLR3, TLR4, and TLR9, and suggest the potential use of TLRs in target therapy.
\end{abstract}

\section{Introduction}

Heart failure (HF) is a complex clinical syndrome and occurs during structural or functional impairment of ventricular filling or ejection of blood in the heart that fails to pump sufficiently to maintain blood flow that meets the body's needs. HF is a leading cause of morbidity and mortality worldwide, and it increasingly affects millions of people $[1,2]$. There are three types of HF, the left-sided HF, right-sided HF, and congestive HF, according to the classification of the American Heart Association. There are two kinds of left-sided HF: one is systolic HF, which means that the left ventricle loses its ability to contract normally causing the inability of the heart to pump with enough force to push enough blood into circulation; the other is diastolic HF, which means that the left ventricle loses its ability to relax normally causing the inability of the heart to be properly filled with blood during the resting period between each beat. Right-sided HF usually occurs when the right ventricle loses its ability to be filled with or to inject blood properly. Congestive HF describes the condition that when blood flowing out of the heart is slower than normal, blood returning to the heart through the veins backs up, causing congestion in the body's tissues, including the arms, legs, ankles, feet, and lungs.

HF is caused by many conditions that damage the heart muscle. Ischemic heart disease (IHD) is the number one leading cause of HF according to epidemiological studies. In clinical trials, HF has been ascribed to IHD in about $70 \%$ of patients [3]. Other common causes are involved in the progression of HF, including dilated cardiomyopathy (DCM), cardiomyopathy of an unknown cause, hypertension, atrial fibrillation, infection, excess alcohol use, metabolic syndrome, atherosclerotic disease, myocarditis, and cardiomyopathy due to inflammation $[1,4]$.

Growing evidence supports that inflammation has been implicated in the pathogenesis of HF $[4,5]$. Inflammation 
of the heart may cause HF in about $10 \%$ of cases of initially unexplained cardiomyopathy $[6,7]$. A variety of infectious organisms, as well as toxins and medications, most often postviral in origin, may cause myocarditis. The link between $\mathrm{HF}$ and inflammation was first recognized in 1990 by Levine et al., who reported elevated levels of tumor necrosis factor $\alpha$ $(\mathrm{TNF} \alpha)$ in patients with $\mathrm{HF}$ with reduced ejection fraction [8]. Numerous studies have demonstrated that patients with HF exhibited raised circulating levels of other inflammatory cytokines, such as interleukin- (IL-) $1 \beta$ and IL-6, and several chemokines, including monocyte chemoattractant peptide(MCP-) 1, IL-8, macrophage inflammatory protein- (MIP-) $1 \alpha$, and galectin-3 [9-14]. These data suggest that increased systemic levels of inflammatory cytokines in patients with HF may reflect important pathogenic mechanisms. The systemic metabolic disorders induce subcellular component abnormalities, such as oxidative stress, mitochondrial dysfunction, endoplasmic reticulum (ER) stress, and impaired calcium handling, leading to impaired myocardial relaxation [15]. In addition to myocardium itself, several tissues and cells, including leukocytes, platelets, tissue macrophages, and endothelial cells, can contribute to this inflammation. In the advanced stage, increased subcellular component abnormalities, inflammatory cell infiltration, neurohumoral activation, and their vicious cycle induce cardiomyocyte injury and death and cardiac fibrosis, resulting in impairment of both diastolic and systolic functions, leading to $\mathrm{HF}$ $[15,16]$. In addition, these inflammatory mediators may serve as relevant markers of disease severity and HF prognosis [17-21]. Importantly, the association between $\mathrm{HF}$ and markers of inflammation was observed for both reduced and preserved ejection fraction cohorts [22].

In short, inflammation plays a critical role in myocardial ischemia and the development of HF. More and more evidence is emerging to explore the function of Toll-like receptors (TLRs) in inflammation-caused HF. In this review, we will focus on the roles of TLRs in the progression of HF, updating recent findings.

\section{Pattern Recognition Receptors (PRRs)}

Inflammatory signaling in cardiomyocytes usually occurs as an early response to myocardial injury. Innate immune activation is a key pathogenic mechanism in HF. Mann's study showed the close correlation between innate immunity and HF. Gene arrays from explanted hearts from patients with ischemic cardiomyopathy (ICM), idiopathic DCM, viral cardiomyopathy, and nonfailing hearts show the different expressions of innate immune genes in the failing heart compared to nonfailing hearts, and elevated levels of inflammatory mediators have been identified in patients with a failing heart; this observation indicates the possibility of activation of the innate immune system in failing hearts [11, 23].

Cardiac innate immune responses, which are essential for homeostatic responses and tissue repair, are initiated by germline-encoded PRRs, which include TLRs, retinoic acidinducible gene-I-like receptors, nucleotide-oligomerization domain-like receptors (NLRs), C-type lectin receptors
(CLRs), and absent-in-melanoma 2 receptors [24, 25]. The strategy of PRR recognition is based on the detection of constitutive and conserved pathogen-associated molecular patterns (PAMPs), which include bacterial carbohydrates, nucleic acids, bacterial peptides, peptidoglycans, lipoteichoic acids, $N$-formylmethionine, lipoproteins, fungal glucans, and chitin. PRRs can also recognize endogenous stress signals called damage-associated molecular patterns (DAMPs), including uric acids, extracellular ATP and other compounds [26]. Recently, it has become clear that cardiac PRRs also recognize the molecular patterns of endogenous host material released by dying or injured myocardial cells [11]. Cells that die by accidental necrosis, necroptosis, or secondary apoptosis release their cytosolic contents into the extracellular space, thereby initiating inflammatory responses through engagement of an ensemble of extracellular or intracellular PRRs.

\section{The TLR Signaling Pathway}

TLRs belong to PRRs, which are involved in the inflammatory responses during HF [27]. To date, 13 TLRs have been identified in mammals, with 10 in humans and 12 found in mice; TLRs are type I transmembrane glycoproteins comprising extracellular, transmembrane, and intracellular domains [28, 29]. TLRs are classified into two main groups according to their subcellular localization; TLR1, TLR2, TLR4, TLR5, TLR6, and TLR11 are expressed on the plasma membrane, whereas TLR3, TLR7, TLR8, and TLR9 are found in endosomes [30, 31].

TLRs usually function as dimers for PAMP detection. Individual TLRs differentially recruit members of a set of Toll/IL-1 receptor (TIR) domain-containing adaptors [32]. Five TIR domain-containing adaptors have been identified, namely, myeloid differentiation factor 88 (Myd88), Myd88 adaptor-like protein, TIR domain-containing adaptor protein inducing interferon (IFN)- $\beta$-mediated transcription factor (TRIF), TRIF-related adaptor molecule (TRAM), and a sterile $\alpha$ - and armadillo motif-containing protein $[33,34]$. Based on specific adaptors recruited to TLRs, TLR signaling can be divided into two general pathways, namely, the Myd88-dependent and Myd88-independent pathways. Except for TLR3, all TLRs interact with the adaptor protein Myd88. TLR3 uses TRIF as the adaptor protein belonging to Myd88-independent pathways, whereas TLR4 triggers both the Myd88-dependent and Myd88-independent pathways [35]. These signaling pathways activate numerous transcription factors, such as nuclear factor- $\kappa \mathrm{B}(\mathrm{NF}-\kappa \mathrm{B})$ and interferon (IFN) regulatory factors (IRFs), and subsequently induce the production of proinflammatory cytokines and IFNs, respectively [36].

The Myd88-dependent pathway is initiated via Myd88 after TLR activation [37]. Afterwards, the death domain of Myd88 recruits IL-1 receptor-associated kinase 4 (IRAK4) and activates one of other IRAK family members, that is, IRAK1 or IRAK2. Then, IRAKs dissociate from the My88IRAK complex and activate TNF receptor-associated factor 6 (TRAF6), which interacts with transforming growth factor- $\beta$-activated kinase 1 (TAK1), TAK1-binding protein 1 (TAB1), and TAB2 [33]. TAK1 then activates the complex 


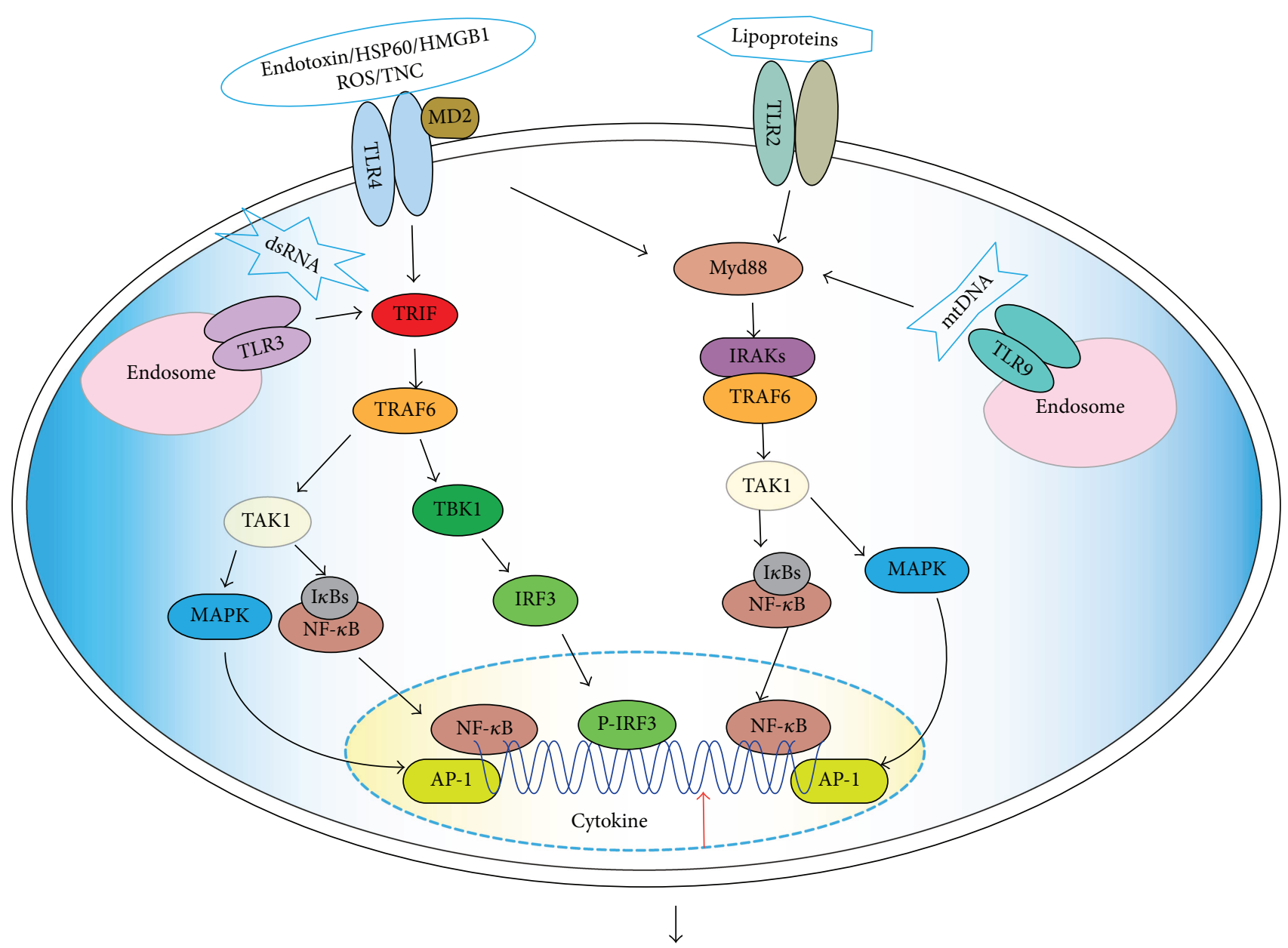

Inflammation

$\downarrow$

Heart failure progression

FIGURE 1: Activation of TLRs in heart cells by PAMPs and DAMPs during heart failure. Heart cells express a variety of TLRs, mainly, TLR2, TLR3, TLR4, and TLR9. DAMP and PAMP molecules, which include endotoxin, HSP60, HMGB1, ROS, TNC, lipoproteins, virus RNA, and mtDNA, are involved in HF. Lipoproteins have been reported to activate TLR2. Endotoxin, HSP60, HMGB1, ROS, and TNC have been demonstrated to activate TLR4. dsRNA can be recognized by TLR3. TLR9 can recognize mtDNA to induce immune responses. There are two pathways for TLR signaling, including the Myd88-dependent and Myd88-independent signaling pathways. TLR2 and TLR9 utilize the Myd88-dependent pathway. TLR3 uses the Myd88-independent pathway. TLR4 employs both Myd88 and TRIF as adaptor proteins. Note that TLR3 and TLR9 are predominately located within endosomes. In the Myd88-dependent signaling pathway, stimulation of TLR triggers association of My88, which in turn recruits the IRAK family, and subsequently, TRAF6 is also recruited to the receptor complex by associating with phosphorylated IRAKs. Ubiquitylation of TRAF6 induces the activation of TAK1, which phosphorylates both MAPK kinases and the IKK complex consisting of IKK- $\alpha$, IKK- $\beta$, and IKK- $\gamma$. The IKK complex then phosphorylates I $\kappa$ B, which is then ubiquitylated and subsequently degraded. This result allows NF- $\kappa \mathrm{B}$ to translocate to the nucleus and induce the expression of its target genes. TRIF play an essential role in the Myd88-independent pathway through TLR3 and TLR4. TRIF interacts with TRAF6, which activates TBK1 and IKK- $\varepsilon$ for phosphorylation of the transcription factor IRFs. TRIF can also promote NF- $\kappa$ B activation. TRIF recruits TRAF6 and activates TAK1, which in turn activates the NF- $\kappa \mathrm{B}$ and MAPK pathways. These signaling pathways result in the expression of cytokines. Inflammation induces cell injury and death, resulting in cardiac dysfunction and HF progression.

of inhibitory $\kappa \mathrm{B}(\mathrm{I} \kappa \mathrm{B})$ kinase $\alpha(\mathrm{IKK} \alpha) / \mathrm{IKK} \beta / \mathrm{IKK} \gamma$ and induces $\mathrm{I} \kappa \mathrm{B}$ phosphorylation. Phosphorylated $\mathrm{I} \kappa \mathrm{B}$ dissociates from the complex and activates the transcription factor NF$\kappa \mathrm{B}$. The activated NF- $\kappa \mathrm{B}$ translocates into the nucleus and induces the expression of various proinflammatory cytokines. In addition to the activation of the IKK complex, TAK1 can activate the mitogen-activated protein kinase
(MAPK) signaling pathway, including the extracellular signal-regulated kinase pathway, c-Jun $\mathrm{N}$-terminal kinase pathway, and p38 pathway. The MAPK signaling pathway can activate the transcription factor activator protein-1 (AP-1). Activation of NF- $\kappa \mathrm{B}$ and AP-1 contributes to the expression of proinflammatory cytokines, such as IL-6, IL-1, and TNF $\alpha$ (Figure 1). 
TABLE 1

\begin{tabular}{lccc}
\hline TLR & Expression & Related pathogenesis of HF & Therapy direction \\
\hline TLR2 & Second highest & $\begin{array}{c}\text { Doxorubicin-induced DCM; MI; contribute to } \\
\text { myocardial inflammation; similar expression } \\
\text { in patients with chronic HF }\end{array}$ & $\begin{array}{c}\text { Inhibition of TLR2 is beneficial for the } \\
\text { progression of HF; blocking molecular } \\
\text { T2.5 antibody }\end{array}$ \\
\hline TLR3 & Third highest & $\begin{array}{c}\text { Protected virus-induced myocardial injury; MI; } \\
\text { contribute to myocardial inflammation }\end{array}$ & Unclear \\
TLR4 & The highest & $\begin{array}{c}\text { Bacteria-induced myocardial injury; virus-induced } \\
\text { myocardial injury; contribute to myocardial inflammation; } \\
\text { doxorubicin-induced DCM; MI; myocardial } \\
\text { ischemia-reperfusion injury; higher expression in } \\
\text { patients with chronic HF }\end{array}$ & $\begin{array}{c}\text { Inhibition of TLR4 is beneficial } \\
\text { for the progression of HF; blocking } \\
\text { molecular such as the statin } \\
\text { family, eritoran, RP105, and ghrelin }\end{array}$ \\
\hline TLR9 & The lowest & $\begin{array}{c}\text { Bacteria-induced myocardial injury; myocarditis; } \\
\text { DCM; contribute to myocardial inflammation; } \\
\text { diastolic HF; elevated mtDNA in HF patients }\end{array}$ & $\begin{array}{c}\text { Inhibition of TLR9 is beneficial for } \\
\text { the progression of HF; pretreatment } \\
\text { with synthetic TLR9 ligand }\end{array}$ \\
\hline
\end{tabular}

Also known as the TRIF-dependent pathway, the Myd88-independent pathway can lead to activation of IRFs and NF- $\kappa$ B. This pathway is initiated by TRIF and TRAM. TRAM is a particular adaptor connecting TLR and TRIF. After recruitment, TRIF interacts with TRAF6, which activates TRAF family member-associated NF- $\kappa \mathrm{B}$ activatorbinding kinase 1 (TBK1) and IKK- $\varepsilon$ for phosphorylation of IRFs. Activated IRFs translocate into the nucleus to induce the production of IFNs. In another type of signaling, TRIF can promote NF- $\kappa$ B activation. TRIF recruits TRAF6 and activates TAK1, which in turn activates NF- $\kappa \mathrm{B}$ and MAPK pathways. Activation of NF- $\kappa \mathrm{B}$ and AP-1 contributes to the expression of proinflammatory cytokines, whereas the activation of IRF3 contributes to the expression of interferon (Figure 1).

\section{TLR Signaling and HF}

Recently, increasing evidence indicated involvement of innate immune activation mediated by myocardial TLRs in HF [12]. TLRs are expressed in various types of heart cells, including endothelial cells, smooth muscle cells, and cardiomyocytes [38]. Relative expression levels for TLR mRNAs in the human heart follow the order TLR4 $>$ TLR2 $>$ TLR $3>$ TLR $5>$ TLR $1>$ TLR6 $>$ TLR7 $>$ TLR8 $>$ TLR9 $>$ TLR10 $[11,39]$. These TLRs not only present different expressions but also perform different functions in the development of HF. Next, we will discuss several important TLRs, including TLR2, TLR3, TLR4, and TLR9, which are associated with HF (Figure 1, Table 1).

4.1. TLR2. TLR2 is located on the cell surface, and along with TLR1 or TLR6, it recognizes a wide variety of PAMPs, including lipoproteins, peptidoglycans, lipoteichoic acids, zymosan, mannan, and mucin [37]. A clinical study shows that there is a possible immunological role for lipoproteins in chronic HF [40].

TLR2 plays a central role in the pathogenesis of diverse heart disorders and is upregulated in doxorubicin-reduced DCM and HF [41]. An early report showed that TLR2 was expressed in cardiomyocytes, was a participant in responses of these cells to oxidative stress, and was a major contributor to the pathogenesis of cardiac dysfunction [42]. Vascular endothelial cells also express high levels of TLR2 on stimulation of inflammatory cytokines, suggesting that TLR2 can also contribute to endothelial cell-related inflammation [43]. In the therapeutic study, Ma et al. demonstrated that blockage of TLR2 reduced mortality and attenuated doxorubicin-induced cardiac dysfunction and inhibition of TLR2 showed a potential role for the treatment of DCM [41].

In a mouse model, TLR2 was involved in cardiac remodeling after myocardial infarction (MI), and preservation of cardiac function, increased survival rate, and attenuation of myocardial fibrosis after MI in TLR2 KO mice were observed [44]. Adverse ventricular remodeling following cardiac injury is a key determinant of HF. Other TLR2 studies were accompanied with TLR4. Activation of TLR2 and TLR4 worsened ischemic injury to the heart and brain of animal models of MI and stroke [45]. A study pointed out that TLR2 and TLR4 influence autonomic regulation of heart rate, and mice lacking TLR2 or TLR4 exhibited reduced basal heart rate [46].

Moreover, increased expression and signaling by TLR2 have been found to contribute to the activation of innate immunity in injured myocardium, indicating that TLR2 can promote myocardial inflammation in HF [44]; however, another study indicated that TLR2 expressions in patients with chronic HF are similar compared with that in the control group [47]. In conclusion, evidence is shown that inhibition of TLR2 reduces the progression of HF (Table 1).

In terms of inhibiting TLR2 signaling, antibodies are designed. T2.5 is one of the anti-TLR2 antibodies with therapeutic potential. Moreover, T2.5 was found to prevent angiotensin II-induced cardiac fibrosis through suppressing macrophage recruitment and inflammation in the heart [48].

4.2. TLR3. TLR3 is localized in endosomes and recognizes viral double-stranded RNA (dsRNA), small interfering RNA, and self-RNA that is derived from damaged cells [49].

Few studies have discussed virus-caused inflammation related to TLR3 in HF. Enterovirus-induced myocardial injury can lead to severe HF. TLR3 plays an important role in the initiation of innate antiviral responses. Hardarson et al. [50] reported that TLR3 KO mice were more 
susceptible for encephalomyocarditis virus infection and featured a higher viral load in the heart and that TLR3 was involved in mediating protection against virus-induced myocardial injury.

Another research by Wang et al. reported that TLR3-deficient neonatal hearts exhibited impaired cardiac functions and larger infarct size after MI compared to control, which indicated that TLR3 is also related with HF [51]. Similarly, Chen et al. reported that TLR3 signaling was involved in MI and extracellular RNA released during myocardial ischemia-reperfusion (I/R) injury, which may contribute to myocardial inflammation [52].

TLR3 primarily protects the heart against viral infection; however, TLR3 also mediates inflammatory effects that may exacerbate heart damage (Table 1).

4.3. TLR4. All known human TLRs have been detected in the heart and most importantly, TLR4, whose levels are the highest compared with other TLRs in the heart [23]. TLR4 plays a critical role in myocardial inflammation, including myocarditis, MI, myocardial I/R injury, HF, aortic valve diseases, atherosclerosis, and hypertension [27, 53].

TLR4 is located at the plasma membrane, where it responds to its ligands and triggers a series of inflammatory signaling pathways [54]. TLR4 is activated by lipopolysaccharide (LPS), with the cofactors, such as cluster of differentiation 14, myeloid differentiation factor 2 (MD2), and lipopolysaccharide- (LPS-) binding protein [55, 56]. Increased levels of bacterial LPS have been demonstrated in HF [57]. Endotoxin is an LPS constituent of the outer membrane of most Gram-negative bacteria. The endotoxin can bind to TLR4/MD2 complexes, which cause subsequent inflammation, and has been implicated in the development and progression of atherosclerosis and subsequent coronary artery disease and HF [58]. Another report also showed that the cardiac function in TLR4-deficient mice was not affected following septic shock or myocardial ischemia [59].

TLR4 can also recognize exogenous ligands, such as the fusion proteins from respiratory syncytial virus and glycerophosphatidylinositol anchors from parasites [31]. In a mouse model, Riad et al. [60] reported that coxsackievirus infection with TRIF-deficient mice can lead to the induction of severe $\mathrm{HF}$ and $100 \%$ mortality, displaying TLR4-dependent suppression of antiviral cytokine IFN- $\beta$. By contrast, coxsackievirus infection increased the cardiac levels of IL- $1 \beta$ and IL-18 in WT mice but not in TLR4deficient ones, and TLR4 deletion may protect these animals from HF [61]. From a perspective, it is likely that the role of TLR4 is indistinct with regard to protecting the heart against viruses.

Some endogenous ligands, such as heat shock protein (HSP), high-mobility group box 1 (HMGB1), reactive oxygen species (ROS), and extracellular matrix components, can be recognized by TLR4 $[31,62]$. Some of these ligands are associated with HF. HSP60 is doubly expressed in end-stage HF and presents abnormal trafficking to the cell surface, which may be an early trigger for myocyte loss and the progression of HF [63]. HMGB1 has been established as an important mediator of myocardial inflammation and is associated with the progression of HF. The study of Volz et al. [64] showed that HMGB1 plasma concentration was elevated in HF and correlated with disease severity in patients with HF. ROS can modify membrane components and can cause the release of factors that interact with and activate TLR4 to induce cardiomyocyte apoptosis and HF $[10,65]$. Tenascins represent a family of four multimeric extracellular matrix glycoproteins [66]. Serum level of tenascin C (TNC) correlates with the severity of HF [67]. Maqbool et al. [68] reported that TNC can stimulate TLR4 to upregulate the expression of IL-6, contributing to the worsening and progression of HF.

Doxorubicin-induced systemic inflammation is driven by upregulation of TLR4 and endotoxin leakage [69]. TLR4 is upregulated in doxorubicin-induced DCM and HF like TLR2. But unlike the role of TLR2, Ma et al. showed that TLR4 played a distinct function in the progression of doxorubicin-induced DCM and blockage of TLR4exacerbated cardiac dysfunction and fibrosis by amplifying inflammation [41].

Liu et al. [70] pointed that the expression, ligand-binding capacity, and proinflammatory function of TLR4 were upregulated in the cardiomyocytes isolated from the long-term MI, promoting inflammation and exacerbating HF. TLR4 not only is expressed in cardiomyocytes but is also a major feature of activated monocytes and substantially increases in response to DAMPs. Similar to TLR2, TLR4 was expressed at high levels in vascular endothelial cells; this finding suggests contribution of endothelial cell-related inflammation [43]. Peripheral monocytosis may affect the development of $\mathrm{HF}$ after acute MI (AMI). Activated TLR4 in monocytes plays an important role in the synthesis of proinflammatory cytokines. Activation of TLR4 through Cardiomyocytic inflammatory reaction was associated with HF after AMI [26].

TLR4 also has a proinflammatory role in murine myocardial I/R injury. In one study, TLR4-deficient mice sustained smaller infarctions and exhibited less inflammation after myocardial I/R injury [71]. Another study showed that inhibition of TLR4 in an in situ murine model significantly reduced I/R injury and markers of inflammatory response [72].

Studies have shown that TLR4 expression increases in the hearts of patients with advanced $\operatorname{HF}[73,74]$. Other studies indicated that unstimulated monocyte TLR4 expression was significantly higher in patients with chronic HF compared to controls and upregulation of monocyte TLR4 may contribute to pathophysiology of chronic HF [47]. TLR4 is associated with deleterious inflammatory effects that exacerbate heart damage, and inhibition of TLR4 reduces the progression of HF (Table 1).

Pharmacological blocking of TLR4 by different molecules is influenced. Statins are among the early-developed drugs with newly discovered inhibitory activity on TLR4 signaling. Among the statin family, fluvastatin, simvastatin, and atorvastatin, all have shown potent inhibitory activity on TLR4 and subsequent inflammatory pathways to reduce inflammation in vascular systems [48]. Another molecule eritoran, the antagonist of TLR4, is very helpful. Inhibition of TLR4 with eritoran can attenuate myocardial ischemiareperfusion injury [75] and the development of cardiac 
hypertrophy [76]. There is a new finding of radioprotective $105 \mathrm{kDa}$ protein RP105, which is a regulator of TLR4 and critical therapeutic target, which can protect against I/R injury via suppressing TLR4 signaling pathways in a rat model [77, 78]. Ghrelin is another candidate for suppression of TLR4 signaling and has protective effects against inflammation in a mouse model of myocardial $\mathrm{I} / \mathrm{R}$ injury via the TLR4 pathway [79].

4.4. TLR9. TLR9 was first identified as a TLR-recognizing cytosine-phosphate-guanine $(\mathrm{CpG})$ which repeats within microbial DNA and is expressed in the myocardium [80, 81]. TLR9 mainly signals through the Myd88-dependent pathway and stimulates NF- $\kappa \mathrm{B}$ and downstream signaling. Emerging evidence has shown the involvement of TLR9 in HF.

Stimulation with bacterial DNA or CpG-rich DNA can induce myocardial inflammation and reduce cardiomyocyte contractility through TLR9 [82]. Another research on a mouse model with polymicrobial sepsis indicated that TLR9 KO mice showed significant reduction in cardiac inflammation and sustained heart function, indicating that TLR9 promotes cardiac inflammation and HF during polymicrobial sepsis [83].

Mitochondrial DNA (mtDNA) is similar to bacterial DNA and may contain high contents of $\mathrm{CpG}$ that activates TLR9 $[84,85]$. Recent data has demonstrated that mtDNA was a DAMP that activated TLR9 [86-88]. Extracellular mtDNA activates NF- $\kappa$ B via TLR9 and can induce cell death of cardiomyocytes [89]. Pathophysiological significance of TLR9 in HF has been studied. Oka et al. [86] studied that the mtDNA that escaped from autophagy cell autonomously leads to TLR9-mediated inflammatory responses in cardiomyocytes and can induce myocarditis and DCM. DNase II is an acid DNase found in lysosomes and plays an important role in preventing pressure overload-induced HF. Inhibition or ablation of TLR9 attenuated the development of cardiomyopathy in DNase II-deficient mice. TLR9 KO mice showed improved pressure overload-induced cardiac dysfunction and inflammation [86]. On the contrary, Velten et al. [90] indicated that pretreatment with synthetic TLR9 ligand 1668-thioate attenuated cardiac hypertrophy following pressure overload and delayed the cardiac function loss, which resulted in a prolonged preservation of left ventricular function. A similar study showed another synthetic agonist of TLR9 that activated the phosphoinositide 3-kinase/protein kinase B signaling pathway and attenuated pathological cardiac hypertrophy and HF [91]. All these data have shown that altered TLR9 signaling influences the progression of HF although the results reflect some differences in experimental models.

Other studies showed that TLR9 plays an important role in diastolic HF. The sacro/endoplasmic reticulum $\mathrm{Ca}^{2+}$ ATPase (SERCA) is the nodal protein that governs active diastolic function [92]. Cardiomyocyte-specific deletion of SERCA2a leads to diastolic HF [93]. Dhondup et al. [94] reported that in a mouse model with diastolic HF caused by cardiomyocyte-specific deletion of SERCA2a, sustained activation of TLR9 caused cardiac and systemic inflammation and deterioration of SERCA2a depletion-mediated diastolic HF. In another diastolic HF mouse model induced by cardiomyocyte-specific deletion of SERCA2a, TLR9 depletion in those models reduced the survival rate compared with that of the SERCA2a KO control mice; this finding indicates the salutary role of TLR9 in some subsets of HF [95]. These studies suggest a link between systemic TLR9 activation and diastolic HF.

In a clinical study, Ye et al. [96] discovered increased plasma-derived exosomes in patients with chronic HF compared with healthy controls and demonstrated that plasma-derived exosomes carry mtDNA, which can trigger an inflammatory response via the TLR9-mediated NF- $\kappa$ B pathway. Another study showed elevated plasma levels of mtDNA from patients with HF, and this condition was associated with increased mortality [97]. Interestingly, TLR9 is only expressed to a small amount in the human heart compared with other TLRs $[11,39]$ but does seem to play an important role in HF. This is a little contradiction. Actually, mitochondria are recognized as a key player in cardiomyocyte cell death after myocardial infarction and cardiomyopathies. TLR9 is very important for the recognition of mtDNA in mitochondria, and that may be the key point. These findings indicate that the TLR9 signaling pathway is involved in inflammatory responses and the pathogenesis of HF (Table 1).

\subsection{The Downstream Molecular Pathway of TLR Signaling.} TLR signaling pathway downstream molecules are involved in the HF progression. Myd88 is central to the signaling of most of the TLRs and receptors of the IL-1 family. One study pointed out that Myd88 deletion can protect mice from the progression of acute myocarditis to end-stage HF [98]. Other two studies showed that Myd88-mediated inflammatory signaling leads to CaMKII oxidation, cardiac hypertrophy, and death after MI and blockade of Myd88 with ST2825 or IMG2005 prevents left ventricular dilation and hypertrophy after acute MI $[99,100]$. Myd88 could recruit the IRAK family member IRAK4, and deletion of IRAK4 has favorable effects on survival and left ventricular remodeling after MI [101]. Negative regulation of inflammatory signaling involves activation of distinct pathways in various cell types involved in cardiac repair. IRAK-M exerts its antiinflammatory actions by inhibiting TLR/IRAK-1-dependent signaling in macrophages. Genetic IRAK-M loss was associated with accentuated inflammation and increased dilative remodeling following infarction [102, 103]. The IKK and its downstream target, NF- $\kappa \mathrm{B}$, are regulators of inflammation and are activated in cardiac disorders [104, 105]. Maier et al.'s study showed that cardiomyocytespecific IKK/NF- $\kappa$ B activation induced reversible inflammatory cardiomyopathy and HF [106]. Frantz et al.'s study pointed out that deletion of the NF- $\kappa \mathrm{B}$ subunit p50 in mouse improved early survival and reduces left ventricular dilatation after MI; these findings indicate that NF- $\kappa \mathrm{B}$ may therefore be an attractive target for HF treatment [107]. The chemokine MCP-1, a downstream molecule of TLR signaling, has been considered as one of the biomarkers of HF [108]. 


\section{Other PRRs and HF}

NLRs act as cytosolic sensors to intracellular PAMPs and DAMPs. The human NLR family includes 22 members, most of which share a conserved tripartite structure consisting of an $\mathrm{N}$-terminal caspase recruitment domain (CARD) or pyrin domain, a central nucleotide-binding domain with NTPase activity, and a C-terminal leucine-rich repeat domain that mediates ligand sensing [109]. NLRP3 belongs to the NLR family, together with apoptosis-associated speck-like protein containing a CARD protein, and forms the NLRP3 inflammasome. This inflammasome represents a complex of intracellular interaction proteins that trigger maturation of proinflammatory cytokines IL- $1 \beta$ and IL- 18 by caspase- 1 to initiate the inflammatory responses $[110,111]$. The NLRP3 inflammasome signaling effector, caspase-1, is upregulated in murine and human failing hearts [112]. NLRP3 KO in cardiac-specific calcineurin transgenic mice resulted in DCM, reduced proinflammatory cytokine maturation and cardiac inflammation, and improved systolic performance [110]. TLR signaling is important for inflammasome priming, and without priming, NLRP3 activation may be insufficient for inducing cardiac dysfunction $[113,114]$. NLRP3 inhibition has been shown to be protective for cardiac function after ischemic injury (AMI) and nonischemic injury (doxorubicin treatment) in mice [115]. Downstream proinflammatory cytokine IL-18 is being considered as a therapeutic target in acute MI and HF [116]. The Canakinumab Anti-inflammatory Thrombosis Outcomes Study (CANTOS) trial, using a blocker of IL-1 $\beta$, has shown good results for the anti-inflammatory therapies in AMI and HF [117]. All these data show that NLRP3 inflammasome signals play an important role in modulating inflammation that affects HF progression. Another NLR protein, NLR family CARD domain-containing protein 4 (NLRC4), has been observed in heart diseases, and this study showed that NLRC4 inflammasome was hyperactivated by mitochondrial DNA in cardiomyocytes in a type 2 diabetes mouse model after MI [118].

CLRs are calcium-dependent carbohydrate-binding receptors that contain one or more C-type lectin-like domains. CLRs form a large family that recognizes a diverse array of structurally unrelated molecules. CLRs and CLRrelated signaling molecules are constitutively expressed in human and murine hearts [119]. Expressions of CLRs and CLR-related signaling molecules in a healthy heart support the possible expression of CLRs in cardiomyocytes; however, additional work is needed to fully define the functions of these proteins.

\section{Conclusion and Prospective}

Inflammation has been widely accepted to play an important role in the physiological and pathological mechanisms of cardiac function and dysfunction. Inflammation is required for host defense against damage and tissue repair. However, excessive chronic myocardial inflammation is reported to induce severe damage to the myocardium and cause HF. The role of the innate immune system in the pathogenesis of heart diseases has been an area of particular focus; targeting innate immune molecules in experimental models can variously attenuate disease progression and injury and promote healing [12].

In this review, we described some recent advances in our understanding of the role of TLR receptors in HF. The provided data linking TLR signaling to HF is still being accumulated at the time of writing. TLR signaling pathway modulates much broader regulation of inflammatory mediators and acts as an important upstream mechanism for activating inflammatory signaling. Hence, target of TLR singnaling molecular in HF may offer a reliable therapeutic approach. Accordingly, various therapeutic agents for inhibiting TLR signaling have been developed to control excessive inflammation [48].

However, few issues remain unanswered. For instance, (1) the mechanism of TLR activation remains unelucidated. More ligands must still be identified in the failing heart; (2) regulation of TLRs in the failing heart also requires further research. Better understanding of these questions will potentially generate a novel therapy for preventing or slowing of the development and progression of HF. Moreover, targeting specific TLR pathways may supply smart strategies for patients with HF. However, at present, our knowledge of the role of TLR signaling is still too insufficient to support the evaluation of this therapy in clinical trials. Although in some models, the role of TLRs is to protect the heart, the expression of TLRs within the heart is often associated with inflammation that leads to increased cell apoptosis, cell necrosis, and tissue damage. On the other side, whether blocking these TLR receptors with antibodies or small molecule inhibitors could prevent the development of a heart failure phenotype needs further identification in animal models.

\section{Conflicts of Interest}

These authors have no conflicts to declare.

\section{Acknowledgments}

This work was supported by the China Scholarship Council (CSC), National Natural Science Foundation of China (Grant no. 81500675), and Doctoral Program of Xinxiang Medical University (Grant no. 2015505084).

\section{References}

[1] C. W. Yancy, M. Jessup, B. Bozkurt et al., "2013 ACCF/AHA guideline for the management of heart failure: a report of the American College of Cardiology Foundation/American Heart Association Task Force on practice guidelines," Circulation, vol. 128, no. 16, pp. e240-e327, 2013.

[2] "Correction to: 2016 ACC/AHA/HFSA focused update on new pharmacological therapy for heart failure: an update of the 2013 ACCF/AHA guideline for the management of heart failure: a report of the American College of Cardiology Foundation/American Heart Association Task Force on clinical practice guidelines and the Heart Failure Society of America," Circulation, vol. 134, no. 13, p. e298, 2016. 
[3] J. G. Cleland and J. McGowan, "Heart failure due to ischaemic heart disease: epidemiology, pathophysiology and progression," Journal of Cardiovascular Pharmacology, vol. 33, Supplement 3, pp. S17-S29, 1999.

[4] H. Nakayama and K. Otsu, "Translation of hemodynamic stress to sterile inflammation in the heart," Trends in Endocrinology and Metabolism, vol. 24, no. 11, pp. 546-553, 2013.

[5] A. Yndestad, J. K. Damas, E. Oie, T. Ueland, L. Gullestad, and P. Aukrust, "Systemic inflammation in heart failure - the whys and wherefores," Heart Failure Reviews, vol. 11, no. 1, pp. 83-92, 2006.

[6] I. Kindermann, C. Barth, F. Mahfoud et al., "Update on myocarditis," Journal of the American College of Cardiology, vol. 59, no. 9, pp. 779-792, 2012.

[7] P. Richardson, W. McKenna, M. Bristow, B. Maisch, and B. Mautner, "Report of the 1995 World Health Organization/International Society and Federation of Cardiology Task Force on the definition and classification of cardiomyopathies," Circulation, vol. 93, no. 5, pp. 841-842, 1996.

[8] B. Levine, J. Kalman, L. Mayer, H. M. Fillit, and M. Packer, "Elevated circulating levels of tumor necrosis factor in severe chronic heart failure," New England Journal of Medicine, vol. 323, no. 4, pp. 236-241, 1990.

[9] K. Nishida and K. Otsu, "Sterile inflammation and degradation systems in heart failure," Circulation Journal, vol. 81, no. 5, pp. 622-628, 2017.

[10] L. Bellumkonda, D. Tyrrell, S. L. Hummel, and D. R. Goldstein, "Pathophysiology of heart failure and frailty: a common inflammatory origin?" Aging Cell, vol. 16, no. 3, pp. 444-450, 2017.

[11] D. L. Mann, "Innate immunity and the failing heart: the cytokine hypothesis revisited," Circulation Research, vol. 116, no. 7, pp. 1254-1268, 2015.

[12] V. K. Topkara, S. Evans, W. Zhang et al., "Therapeutic targeting of innate immunity in the failing heart," Journal of Molecular and Cellular Cardiology, vol. 51, no. 4, pp. 594-599, 2011.

[13] B. Bozkurt, D. L. Mann, and A. Deswal, "Biomarkers of inflammation in heart failure," Heart Failure Reviews, vol. 15, no. 4, pp. 331-341, 2010.

[14] E. A. Medvedeva, I. I. Berezin, E. A. Surkova, D. M. Yaranov, and Y. V. Shchukin, "Galectin-3 in patients with chronic heart failure: association with oxidative stress, inflammation, renal dysfunction and prognosis," Minerva Cardioangiologica, vol. 64, no. 6, pp. 595-602, 2016.

[15] K. Nishida and K. Otsu, "Inflammation and metabolic cardiomyopathy," Cardiovascular Research, vol. 113, no. 4, pp. 389-398, 2017.

[16] M. Diamant, H. J. Lamb, J. W. Smit, A. de Roos, and R. J. Heine, "Diabetic cardiomyopathy in uncomplicated type 2 diabetes is associated with the metabolic syndrome and systemic inflammation," Diabetologia, vol. 48 , no. 8, pp. 16691670,2005

[17] B. W. Van Tassell, R. A. Arena, S. Toldo et al., "Enhanced interleukin-1 activity contributes to exercise intolerance in patients with systolic heart failure," PLoS One, vol. 7, no. 3, article e33438, 2012.

[18] L. Gullestad, T. Ueland, L. E. Vinge, A. Finsen, A. Yndestad, and P. Aukrust, "Inflammatory cytokines in heart failure: mediators and markers," Cardiology, vol. 122, no. 1, pp. 23$35,2012$.
[19] D. L. Mann, "Inflammatory mediators and the failing heart: past, present, and the foreseeable future," Circulation Research, vol. 91, no. 11, pp. 988-998, 2002.

[20] E. Braunwald, "Biomarkers in heart failure," The New England Journal of Medicine, vol. 358, no. 20, pp. 21482159, 2008.

[21] J. P. Rocchiccioli, J. J. McMurray, and A. F. Dominiczak, "Biomarkers in heart failure: a clinical review," Heart Failure Reviews, vol. 15, no. 4, pp. 251-273, 2010.

[22] P. Heidenreich, "Inflammation and heart failure: therapeutic or diagnostic opportunity?," Journal of the American College of Cardiology, vol. 69, no. 10, pp. 1286-1287, 2017.

[23] D. L. Mann, "The emerging role of innate immunity in the heart and vascular system: for whom the cell tolls," Circulation Research, vol. 108, no. 9, pp. 1133-1145, 2011.

[24] E. Kugelberg, "Pattern recognition receptors: curbing gut inflammation," Nature Reviews Immunology, vol. 14, no. 9, p. $583,2014$.

[25] O. Takeuchi and S. Akira, "Pattern recognition receptors and inflammation," Cell, vol. 140, no. 6, pp. 805-820, 2010.

[26] M. Satoh, Y. Shimoda, C. Maesawa et al., "Activated toll-like receptor 4 in monocytes is associated with heart failure after acute myocardial infarction," International Journal of Cardiology, vol. 109, no. 2, pp. 226-234, 2006.

[27] K. Edfeldt, J. Swedenborg, G. K. Hansson, and Z. Q. Yan, "Expression of toll-like receptors in human atherosclerotic lesions: a possible pathway for plaque activation," Circulation, vol. 105, no. 10, pp. 1158-1161, 2002.

[28] L. A. O'Neill, D. Golenbock, and A. G. Bowie, "The history of toll-like receptors - redefining innate immunity," Nature Reviews Immunology, vol. 13, no. 6, pp. 453-460, 2013.

[29] A. L. Blasius and B. Beutler, "Intracellular toll-like receptors," Immunity, vol. 32, no. 3, pp. 305-315, 2010.

[30] Y. Delneste, C. Beauvillain, and P. Jeannin, "Innate immunity: structure and function of TLRs," Medical Science, vol. 23, no. 1, pp. 67-74, 2007.

[31] K. Takeda and S. Akira, "Toll-like receptors in innate immunity," International Immunology, vol. 17, no. 1, pp. 1-14, 2005.

[32] T. Kawasaki and T. Kawai, "Toll-like receptor signaling pathways," Frontiers in Immunology, vol. 5, p. 461, 2014.

[33] K. H. Lim and L. M. Staudt, "Toll-like receptor signaling," Cold Spring Harbor Perspective in Biology, vol. 5, no. 1, article a011247, 2013.

[34] K. A. Jenkins and A. Mansell, "TIR-containing adaptors in toll-like receptor signalling," Cytokine, vol. 49, no. 3, pp. 237-244, 2010.

[35] T. Kawai and S. Akira, "Toll-like receptors and their crosstalk with other innate receptors in infection and immunity," Immunity, vol. 34, no. 5, pp. 637-650, 2011.

[36] H. Kumar, T. Kawai, and S. Akira, "Toll-like receptors and innate immunity," Biochemical and Biophysical Research Communications, vol. 388, no. 4, pp. 621-625, 2009.

[37] T. Kawai and S. Akira, "The role of pattern-recognition receptors in innate immunity: update on toll-like receptors," Nature Immunology, vol. 11, no. 5, pp. 373-384, 2010.

[38] J. A. Mitchell, B. Ryffel, V. F. J. Quesniaux, N. Cartwright, and M. Paul-Clark, "Role of pattern-recognition receptors in cardiovascular health and disease," Biochemical Society Transactions, vol. 35, no. 6, pp. 1449-1452, 2007. 
[39] M. Nishimura and S. Naito, "Tissue-specific mRNA expression profiles of human toll-like receptors and related genes," Biological \& Pharmaceutical Bulletin, vol. 28, no. 5, pp. 886892, 2005.

[40] M. Rauchhaus, V. Koloczek, H. Volk et al., "Inflammatory cytokines and the possible immunological role for lipoproteins in chronic heart failure," International Journal of Cardiology, vol. 76, no. 2-3, pp. 125-133, 2000.

[41] Y. Ma, X. Zhang, H. Bao et al., "Toll-like receptor (TLR) 2 and TLR4 differentially regulate doxorubicin induced cardiomyopathy in mice," PLoS One, vol. 7, no. 10, article e40763, 2012.

[42] S. Frantz, R. A. Kelly, and T. Bourcier, "Role of TLR-2 in the activation of nuclear factor $\kappa \mathrm{B}$ by oxidative stress in cardiac myocytes," The Journal of Biological Chemistry, vol. 276, no. 7, pp. 5197-5203, 2001.

[43] A. Yndestad, J. K. Damas, E. Oie, T. Ueland, L. Gullestad, and P. Aukrust, "Role of inflammation in the progression of heart failure," Current Cardiology Reports, vol. 9, no. 3, pp. 236241, 2007.

[44] T. Shishido, N. Nozaki, S. Yamaguchi et al., "Toll-like receptor-2 modulates ventricular remodeling after myocardial infarction," Circulation, vol. 108, no. 23, pp. 2905-2910, 2003.

[45] F. Arslan, B. Keogh, P. McGuirk, and A. E. Parker, "TLR2 and TLR4 in ischemia reperfusion injury," Mediators of Inflammation, vol. 2010, Article ID 704202, 8 pages, 2010.

[46] E. Okun, K. J. Griffioen, S. Rothman et al., "Toll-like receptors 2 and 4 modulate autonomic control of heart rate and energy metabolism," Brain, Behavior, and Immunity, vol. 36, pp. 90100, 2014.

[47] G. Földes, S. von Haehling, D. O. Okonko, E. A. Jankowska, P. A. Poole-Wilson, and S. D. Anker, "Fluvastatin reduces increased blood monocyte toll-like receptor 4 expression in whole blood from patients with chronic heart failure," International Journal of Cardiology, vol. 124, no. 1, pp. 8085, 2008.

[48] W. Gao, Y. Xiong, Q. Li, and H. Yang, "Inhibition of toll-like receptor signaling as a promising therapy for inflammatory diseases: a journey from molecular to nano therapeutics," Frontiers in Physiology, vol. 8, p. 508, 2017.

[49] J. J. Bernard, C. Cowing-Zitron, T. Nakatsuji et al., "Ultraviolet radiation damages self noncoding RNA and is detected by TLR3," Nature Medicine, vol. 18, no. 8, pp. 1286-1290, 2012.

[50] H. S. Hardarson, J. S. Baker, Z. Yang et al., "Toll-like receptor 3 is an essential component of the innate stress response in virus-induced cardiac injury," American Journal of Physiology-Heart and Circulatory Physiology, vol. 292, no. 1, pp. H251-H258, 2007.

[51] X. Wang, T. Ha, J. Kalbfleisch, D. Williams, and C. Li, “TLR3 mediates neonatal heart repair and regeneration through glycolysis-dependent YAP/TAZ mediated miR-152 expression," Circulation, vol. 134, no. 19, 2016.

[52] C. Chen, Y. Feng, L. Zou et al., "Role of extracellular RNA and TLR3-Trif signaling in myocardial ischemia-reperfusion injury," Journal of the American Heart Association, vol. 3, no. 1, article e000683, 2014.

[53] Y. Yang, J. Lv, S. Jiang et al., "The emerging role of toll-like receptor 4 in myocardial inflammation," Cell Death \& Disease, vol. 7, no. 5, article e2234, 2016.
[54] N. J. Gay, M. F. Symmons, M. Gangloff, and C. E. Bryant, "Assembly and localization of toll-like receptor signalling complexes," Nature Reviews. Immunology, vol. 14, no. 8, pp. 546-558, 2014.

[55] A. Płóciennikowska, A. Hromada-Judycka, K. Borzęcka, and K. Kwiatkowska, "Co-operation of TLR4 and raft proteins in LPS-induced pro-inflammatory signaling," Cellular and Molecular Life Sciences, vol. 72, no. 3, pp. 557-581, 2015.

[56] F. Kopp, S. Kupsch, L. Paulowski et al., "Interaction of the LPS-binding protein with host cell membranes: specificity and biological implications," Biophysical Journal, vol. 110, no. 3, pp. 256a-257a, 2016.

[57] J. Niebauer, H. D. Volk, M. Kemp et al., "Endotoxin and immune activation in chronic heart failure: a prospective cohort study," The Lancet, vol. 353, no. 9167, pp. 1838$1842,1999$.

[58] M. A. Horseman, S. Surani, and J. D. Bowman, "Endotoxin, toll-like receptor-4, and atherosclerotic heart disease," Current Cardiology Reviews, vol. 13, no. 2, pp. 86-93, 2017.

[59] R. Fallach, A. Shainberg, O. Avlas et al., "Cardiomyocyte toll-like receptor 4 is involved in heart dysfunction following septic shock or myocardial ischemia," Journal of Molecular and Cellular Cardiology, vol. 48, no. 6, pp. 1236-1244, 2010.

[60] A. Riad, D. Westermann, C. Zietsch et al., "TRIF is a critical survival factor in viral cardiomyopathy," The Journal of Immunology, vol. 186, no. 4, pp. 2561-2570, 2011.

[61] D. Fairweather, S. Yusung, S. Frisancho et al., "IL-12 receptor $\beta 1$ and toll-like receptor 4 increase IL-1 $\beta$ - and IL-18associated myocarditis and coxsackievirus replication," The Journal of Immunology, vol. 170, no. 9, pp. 4731-4737, 2003.

[62] T. Kawai and S. Akira, "The roles of TLRs, RLRs and NLRs in pathogen recognition," International Immunology, vol. 21, no. 4, pp. 317-337, 2009.

[63] L. Lin, S. C. Kim, Y. Wang et al., "HSP60 in heart failure: abnormal distribution and role in cardiac myocyte apoptosis," American Journal of Physiology. Heart and Circulatory Physiology, vol. 293, no. 4, pp. H2238-H2247, 2007.

[64] H. Volz, D. Laohachewin, D. Schellberg et al., "HMGB1 is an independent predictor of death and heart transplantation in heart failure," Clinical Research in Cardiology, vol. 101, no. 6, pp. 427-435, 2012.

[65] Z. W. Liu, H. T. Zhu, K. L. Chen, C. Qiu, K. F. Tang, and X. L. Niu, "Selenium attenuates high glucose-induced ROS/ TLR-4 involved apoptosis of rat cardiomyocyte," Biological Trace Element Research, vol. 156, no. 1-3, pp. 262-270, 2013.

[66] K. Imanaka-Yoshida, "Extracellular matrix remodeling in vascular development and disease," in Etiology and Morphogenesis of Congenital Heart Disease, pp. 221-226, Springer, 2016.

[67] F. Terasaki, H. Okamoto, K. Onishi et al., "Higher serum tenascin- $C$ levels reflect the severity of heart failure, left ventricular dysfunction and remodeling in patients with dilated cardiomyopathy," Circulation Journal, vol. 71, no. 3, pp. 327-330, 2007.

[68] A. Maqbool, E. J. Spary, I. W. Manfield et al., "Tenascin C upregulates interleukin- 6 expression in human cardiac myofibroblasts via toll-like receptor 4," World Journal of Cardiology, vol. 8, no. 5, pp. 340-350, 2016.

[69] L. Wang, Q. Chen, H. Qi et al., "Doxorubicin-induced systemic inflammation is driven by upregulation of toll-like 
receptor TLR4 and endotoxin leakage," Cancer Research, vol. 76, no. 22, pp. 6631-6642, 2016.

[70] L. Liu, Y. Wang, Z. Y. Cao et al., "Up-regulated TLR4 in cardiomyocytes exacerbates heart failure after long-term myocardial infarction," Journal of Cellular and Molecular Medicine, vol. 19, no. 12, pp. 2728-2740, 2015.

[71] J. Oyama, C. Blais Jr., X. Liu et al., "Reduced myocardial ischemia-reperfusion injury in toll-like receptor 4-deficient mice," Circulation, vol. 109, no. 6, pp. 784-789, 2004.

[72] H. Stapel, S. C. Kim, S. Osterkamp et al., “Toll-like receptor 4 modulates myocardial ischaemia-reperfusion injury: role of matrix metalloproteinases," European Journal of Heart Failure, vol. 8, no. 7, pp. 665-672, 2006.

[73] S. Frantz, L. Kobzik, Y. D. Kim et al., "Toll4 (TLR4) expression in cardiac myocytes in normal and failing myocardium," The Journal of Clinical Investigation, vol. 104, no. 3, pp. 271280, 1999.

[74] E. J. Birks, L. E. Felkin, N. R. Banner, A. Khaghani, P. J. Barton, and M. H. Yacoub, "Increased toll-like receptor 4 in the myocardium of patients requiring left ventricular assist devices," The Journal of Heart and Lung Transplantation, vol. 23, no. 2, pp. 228-235, 2004.

[75] A. Shimamoto, A. J. Chong, M. Yada et al., "Inhibition of toll-like receptor 4 with eritoran attenuates myocardial ischemia-reperfusion injury," Circulation, vol. 114, no. 1, pp. I270-I274, 2006.

[76] H. Ehrentraut, C. Weber, S. Ehrentraut et al., "The toll-like receptor 4-antagonist eritoran reduces murine cardiac hypertrophy," European Journal of Heart Failure, vol. 13, no. 6, pp. 602-610, 2011.

[77] X. Li, J. Yang, W. Dong, S. Li, H. Wu, and L. Li, "RP105 protects against myocardial ischemia-reperfusion injury via suppressing TLR4 signaling pathways in rat model," Experimental and Molecular Pathology, vol. 100, no. 2, pp. 281286, 2016.

[78] C. J. Yang, J. Yang, and Z. X. Fan, "Radioprotective $105 \mathrm{kDa}$ protein (RP105): is a critical therapeutic target for alleviating ischemia reperfusion induced myocardial damage via TLR4 signaling pathway," International Journal of Cardiology, vol. 222, pp. 1069-1070, 2016.

[79] N. Sun, H. Wang, and L. Wang, "Protective effects of ghrelin against oxidative stress, inducible nitric oxide synthase and inflammation in a mouse model of myocardial ischemia/ reperfusion injury via the HMGB1 and TLR4/NF- $\kappa$ B pathway," Molecular Medicine Reports, vol. 14, no. 3, pp. 27642770, 2016.

[80] J. H. Boyd, S. Mathur, Y. Wang, R. M. Bateman, and K. R. Walley, "Toll-like receptor stimulation in cardiomyoctes decreases contractility and initiates an NF- $\kappa \mathrm{B}$ dependent inflammatory response," Cardiovascular Research, vol. 72, no. 3, pp. 384-393, 2006.

[81] H. Hemmi, O. Takeuchi, T. Kawai et al., "A toll-like receptor recognizes bacterial DNA," Nature, vol. 408, no. 6813, pp. 740-745, 2000.

[82] P. Knuefermann, M. Schwederski, M. Velten et al., "Bacterial DNA induces myocardial inflammation and reduces cardiomyocyte contractility: role of toll-like receptor 9," Cardiovascular Research, vol. 78, no. 1, pp. 26-35, 2008.

[83] R. Lohner, M. Schwederski, C. Narath et al., "Toll-like receptor 9 promotes cardiac inflammation and heart failure during polymicrobial sepsis," Mediators of Inflammation, vol. 2013, Article ID 261049, 13 pages, 2013.

[84] Y. Pollack, J. Kasir, R. Shemer, S. Metzger, and M. Szyf, "Methylation pattern of mouse mitochondrial DNA," Nucleic Acids Research, vol. 12, no. 12, pp. 4811-4824, 1984.

[85] L. R. Cardon, C. Burge, D. A. Clayton, and S. Karlin, "Pervasive CpG suppression in animal mitochondrial genomes," Proceedings of the National Academy of Sciences of the United States of America, vol. 91, no. 9, pp. 3799-3803, 1994.

[86] T. Oka, S. Hikoso, O. Yamaguchi et al., "Mitochondrial DNA that escapes from autophagy causes inflammation and heart failure," Nature, vol. 485, no. 7397, pp. 251-255, 2012.

[87] Q. Zhang, M. Raoof, Y. Chen et al., "Circulating mitochondrial DAMPs cause inflammatory responses to injury," Nature, vol. 464, no. 7285, pp. 104-107, 2010.

[88] S. Hajizadeh, J. DeGroot, J. M. TeKoppele, A. Tarkowski, and L. V. Collins, "Extracellular mitochondrial DNA and oxidatively damaged DNA in synovial fluid of patients with rheumatoid arthritis," Arthritis Research \& Therapy, vol. 5, no. 5, pp. R234-R240, 2003.

[89] M. Bliksoen, L. H. Mariero, M. K. Torp et al., "Extracellular mtDNA activates NF- $\kappa$ B via toll-like receptor 9 and induces cell death in cardiomyocytes," Basic Research in Cardiology, vol. 111, no. 4, p. 42, 2016.

[90] M. Velten, G. D. Duerr, T. Pessies et al., "Priming with synthetic oligonucleotides attenuates pressure overload-induced inflammation and cardiac hypertrophy in mice," Cardiovascular Research, vol. 96, no. 3, pp. 422-432, 2012.

[91] L. Yang, X. Cai, J. Liu et al., "CpG-ODN attenuates pathological cardiac hypertrophy and heart failure by activation of PI3Ka-Akt signaling," PLoS One, vol. 8, no. 4, article e62373, 2013.

[92] D. M. Bers, "Cardiac sarcoplasmic reticulum calcium leak: basis and roles in cardiac dysfunction," Annual Review of Physiology, vol. 76, no. 1, pp. 107-127, 2014.

[93] W. E. Louch, K. Hougen, H. K. Mork et al., "Sodium accumulation promotes diastolic dysfunction in end-stage heart failure following Serca2 knockout," The Journal of Physiology, vol. 588, no. 3, pp. 465-478, 2010.

[94] Y. Dhondup, I. Sjaastad, H. Scott et al., "Sustained toll-like receptor 9 activation promotes systemic and cardiac inflammation, and aggravates diastolic heart failure in SERCA2a KO mice," PLoS One, vol. 10, no. 10, article e0139715, 2015.

[95] Y. Dhondup, I. Sjaastad, O. Sandanger et al., "Toll-like receptor 9 promotes survival in SERCA2a KO heart failure mice," Mediators of Inflammation, vol. 2017, Article ID 9450439, 11 pages, 2017.

[96] W. Ye, X. Tang, Z. Yang et al., "Plasma-derived exosomes contribute to inflammation via the TLR9-NF- $\kappa$ B pathway in chronic heart failure patients," Molecular Immunology, vol. 87, pp. 114-121, 2017.

[97] Y. Dhondup, T. Ueland, C. P. Dahl et al., "Low circulating levels of mitochondrial and high levels of nuclear DNA predict mortality in chronic heart failure," Journal of Cardiac Failure, vol. 22, no. 10, pp. 823-828, 2016.

[98] P. Blyszczuk, G. Kania, T. Dieterle et al., "Myeloid differentiation factor-88/interleukin-1 signaling controls cardiac fibrosis and heart failure progression in inflammatory dilated cardiomyopathy," Circulation Research, vol. 105, no. 9, pp. 912-920, 2009. 
[99] B. W. Van Tassell, I. M. Seropian, S. Toldo et al., "Pharmacologic inhibition of myeloid differentiation factor 88 (MyD88) prevents left ventricular dilation and hypertrophy after experimental acute myocardial infarction in the mouse," Journal of Cardiovascular Pharmacology, vol. 55, no. 4, pp. 385-390, 2010.

[100] M. V. Singh, P. D. Swaminathan, E. D. Luczak, W. Kutschke, R. M. Weiss, and M. E. Anderson, "MyD88 mediated inflammatory signaling leads to CaMKII oxidation, cardiac hypertrophy and death after myocardial infarction," Journal of Molecular and Cellular Cardiology, vol. 52, no. 5, pp. 11351144, 2012.

[101] Y. Maekawa, N. Mizue, A. Chan et al., "Survival and cardiac remodeling after myocardial infarction are critically dependent on the host innate immune interleukin-1 receptor-associated kinase- 4 signaling: a regulator of bone marrow-derived dendritic cells," Circulation, vol. 120, no. 14, pp. 1401-14, 2009.

[102] N. G. Frangogiannis, "Pathophysiology of myocardial infarction," Comprehensive Physiology, vol. 5, no. 4, pp. 1841-1875, 2015.

[103] P. Christia and N. G. Frangogiannis, "Targeting inflammatory pathways in myocardial infarction," European Journal of Clinical Investigation, vol. 43, no. 9, pp. 986-995, 2013.

[104] R. Dhingra, J. A. Shaw, Y. Aviv, and L. A. Kirshenbaum, "Dichotomous actions of NF- $\kappa \mathrm{B}$ signaling pathways in heart," Journal of Cardiovascular Translational Research, vol. 3, no. 4, pp. 344-354, 2010.

[105] K. Van der Heiden, S. Cuhlmann, L. A. Luong, M. Zakkar, and P. C. Evans, "Role of nuclear factor $\kappa \mathrm{B}$ in cardiovascular health and disease," Clinical Science (London, England), vol. 118, no. 10, pp. 593-605, 2010.

[106] H. J. Maier, T. G. Schips, A. Wietelmann et al., "Cardiomyocyte-specific I $\kappa \mathrm{B}$ kinase (IKK)/NF- $\kappa \mathrm{B}$ activation induces reversible inflammatory cardiomyopathy and heart failure," Proceedings of the National Academy of Sciences, vol. 109, no. 29, pp. 11794-11799, 2012.

[107] S. Frantz, K. Hu, B. Bayer et al., "Absence of NF$\kappa$ Bsubunit p50 improves heart failure after myocardial infarction," The FASEB Journal, vol. 20, no. 11, pp. 1918-1920, 2006.

[108] A. N. Chandrakala, D. Sukul, K. Selvarajan, C. Sai-Sudhakar, B. Sun, and S. Parthasarathy, "Induction of brain natriuretic peptide and monocyte chemotactic protein-1 gene expression by oxidized low-density lipoprotein: relevance to ischemic heart failure," American Journal of Physiology. Cell Physiology, vol. 302, no. 1, pp. C165-C177, 2012.

[109] S. Kesavardhana and T. D. Kanneganti, "Mechanisms governing inflammasome activation, assembly and pyroptosis induction," International Immunology, vol. 29, no. 5, pp. 201-210, 2017.

[110] B. Butts, R. A. Gary, S. B. Dunbar, and J. Butler, "The importance of NLRP3 inflammasome in heart failure," Journal of Cardiac Failure, vol. 21, no. 7, pp. 586-593, 2015.

[111] J. Fuentes-Antras, A. M. Ioan, J. Tunon, J. Egido, and O. Lorenzo, "Activation of toll-like receptors and inflammasome complexes in the diabetic cardiomyopathy-associated inflammation," International Journal of Endocrinology, vol. 2014, Article ID 847827, 10 pages, 2014.

[112] S. Merkle, S. Frantz, M. P. Schon et al., "A role for caspase-1 in heart failure," Circulation Research, vol. 100, no. 5, pp. 645-653, 2007.
[113] S. Toldo, E. Mezzaroma, M. D. McGeough et al., "Independent roles of the priming and the triggering of the NLRP3 inflammasome in the heart," Cardiovascular Research, vol. 105, no. 2, pp. 203-212, 2015.

[114] E. Latz, T. S. Xiao, and A. Stutz, "Activation and regulation of the inflammasomes," Nature Reviews. Immunology, vol. 13, no. 6, pp. 397-411, 2013.

[115] C. Marchetti, S. Toldo, J. Chojnacki et al., "Pharmacologic inhibition of the NLRP3 inflammasome preserves cardiac function after ischemic and nonischemic injury in the mouse," Journal of Cardiovascular Pharmacology, vol. 66, no. 1, pp. 1-8, 2015.

[116] L. C. O’Brien, E. Mezzaroma, B. W. Van Tassell et al., "Interleukin-18 as a therapeutic target in acute myocardial infarction and heart failure," Molecular Medicine, vol. 20, no. 1, p. 1, 2014.

[117] F. Huet, M. Akodad, J. Fauconnier, A. Lacampagne, and F. Roubille, "Anti-inflammatory drugs as promising cardiovascular treatments," Expert Review of Cardiovascular Ther$a p y$, vol. 15, no. 2, pp. 109-125, 2017.

[118] T. Durga Devi, M. Babu, P. Makinen et al., "Aggravated postinfarct heart failure in type 2 diabetes is associated with impaired mitophagy and exaggerated inflammasome activation," The American Journal of Pathology, vol. 187, no. 12, pp. 2659-2673, 2017.

[119] M. Lech, H. E. Susanti, C. Römmele, R. Gröbmayr, R. Günthner, and H.-J. Anders, "Quantitative expression of C-type lectin receptors in humans and mice," International Journal of Molecular Sciences, vol. 13, no. 12, pp. 1011310131, 2012. 


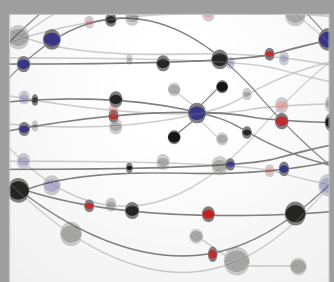

The Scientific World Journal
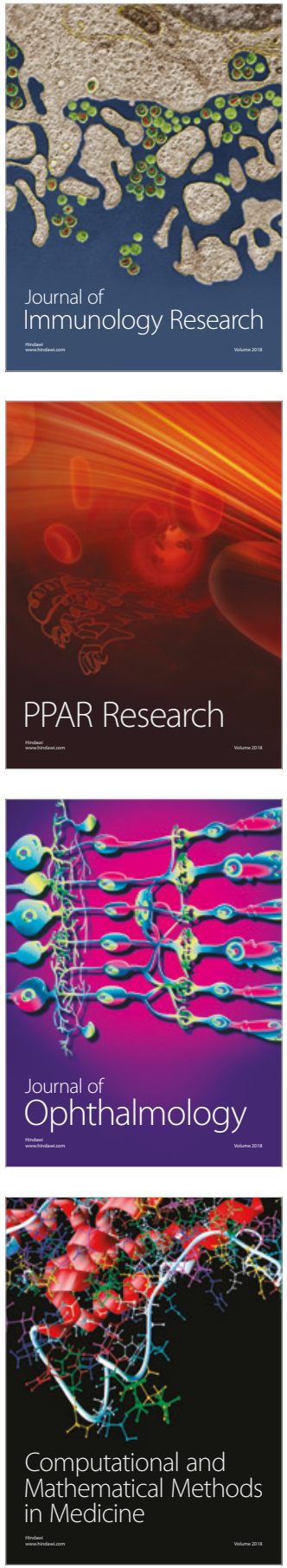

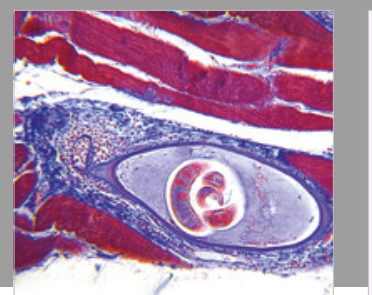

Gastroenterology Research and Practice

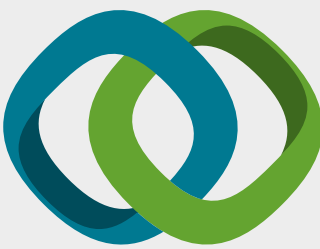

\section{Hindawi}

Submit your manuscripts at

www.hindawi.com
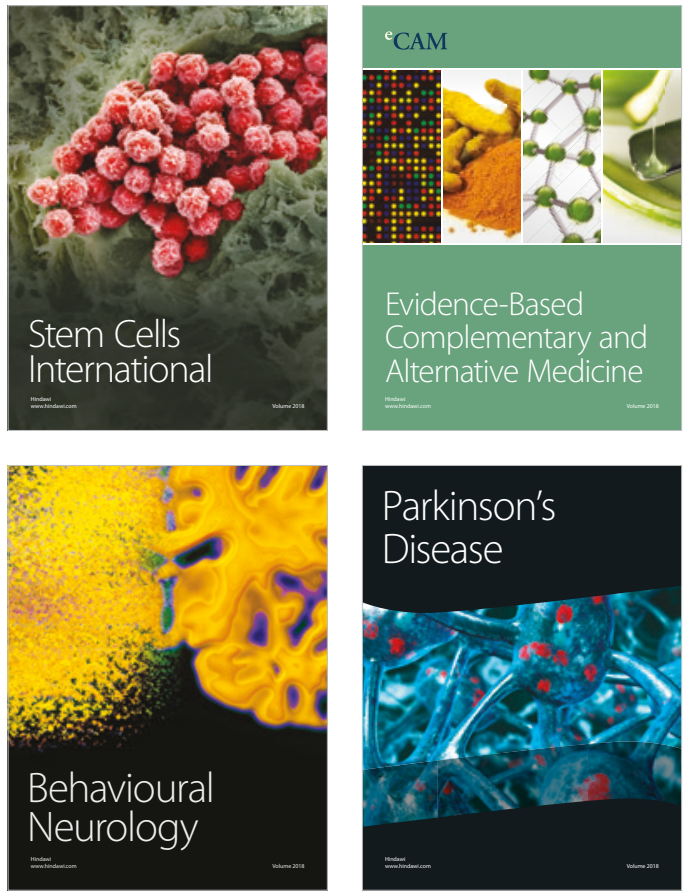

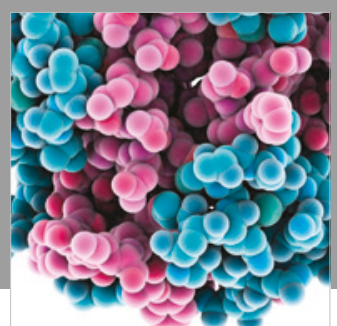

ournal of

Diabetes Research

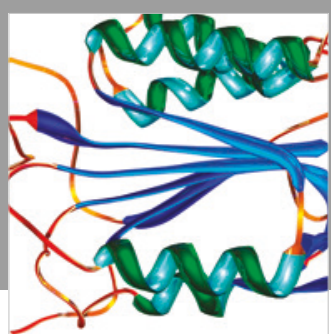

Disease Markers
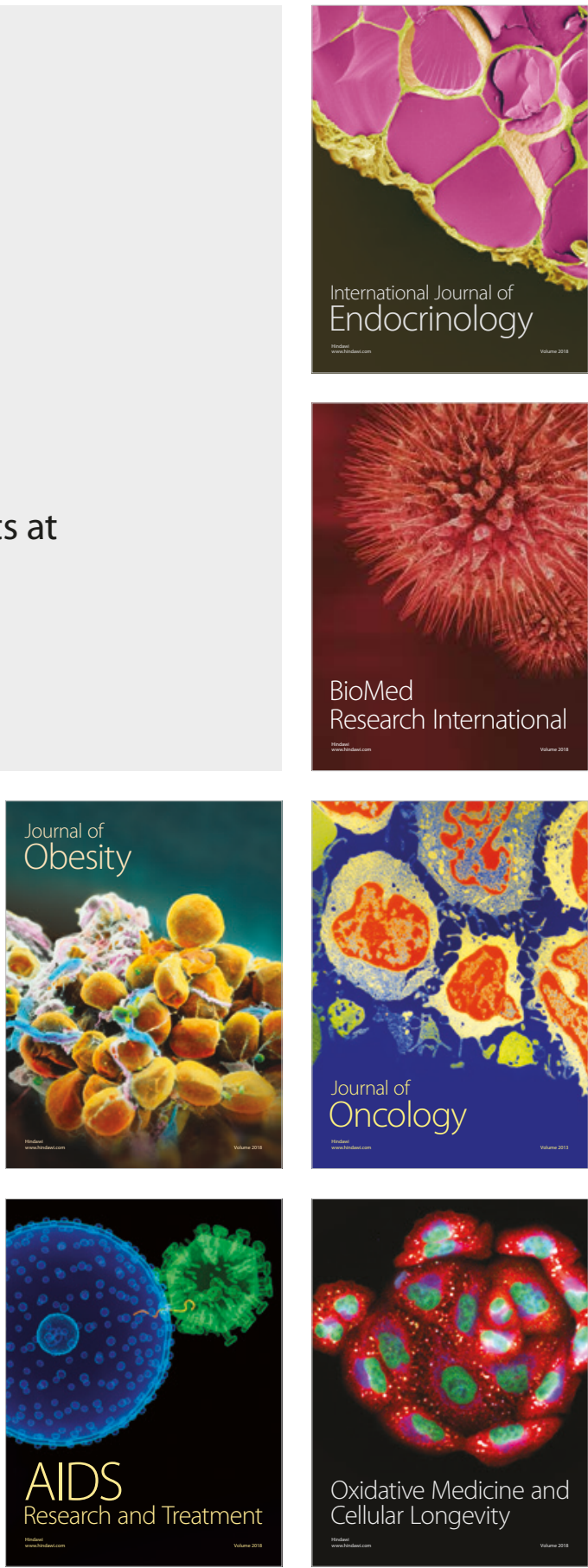